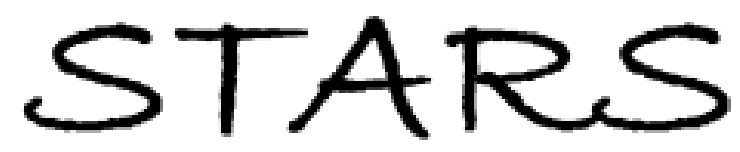

University of Central Florida

STARS

Faculty Bibliography 2000s

Faculty Bibliography

$1-1-2006$

\title{
Crossover in thermal transport mechanism in nanocrystalline silicon
}

Arun Bodapatil

Pawel Keblinski

Patrick K. Schelling

University of Central Florida

Simon R. Philpot

Find similar works at: https://stars.library.ucf.edu/facultybib2000

University of Central Florida Libraries http://library.ucf.edu

This Article is brought to you for free and open access by the Faculty Bibliography at STARS. It has been accepted for inclusion in Faculty Bibliography 2000s by an authorized administrator of STARS. For more information, please contactSTARS@ucf.edu.

\section{Recommended Citation}

Bodapatil, Arun; Keblinski, Pawel; Schelling, Patrick K.; and Philpot, Simon R., "Crossover in thermal transport mechanism in nanocrystalline silicon" (2006). Faculty Bibliography 2000s. 5965.

https://stars.library.ucf.edu/facultybib2000/5965

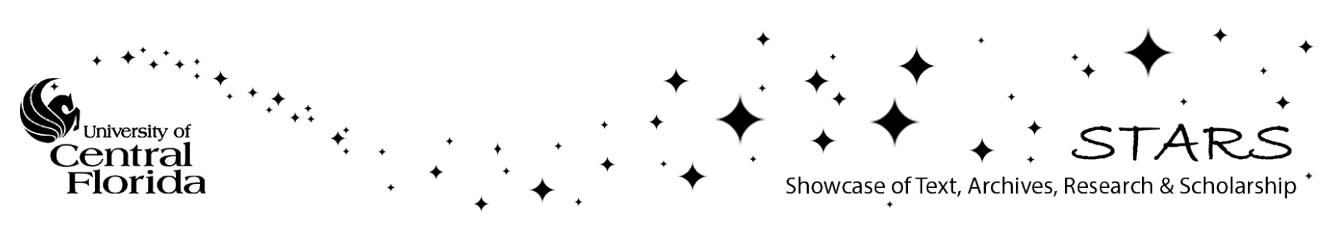




\section{Crossover in thermal transport mechanism in nanocrystalline silicon}

Cite as: Appl. Phys. Lett. 88, 141908 (2006); https://doi.org/10.1063/1.2192145

Submitted: 09 November 2005. Accepted: 07 March 2006 . Published Online: 04 April 2006

Arun Bodapati, Pawel Keblinski, Patrick K. Schelling, and Simon R. Phillpot

\section{ARTICLES YOU MAY BE INTERESTED IN}

Kapitza conductance and phonon scattering at grain boundaries by simulation Journal of Applied Physics 95, 6082 (2004); https://doi.org/10.1063/1.1702100

Phonon wave-packet dynamics at semiconductor interfaces by molecular-dynamics simulation

Applied Physics Letters 80, 2484 (2002); https://doi.org/10.1063/1.1465106

Thermal transport and grain boundary conductance in ultrananocrystalline diamond thin films Journal of Applied Physics 99, 114301 (2006); https://doi.org/10.1063/1.2199974

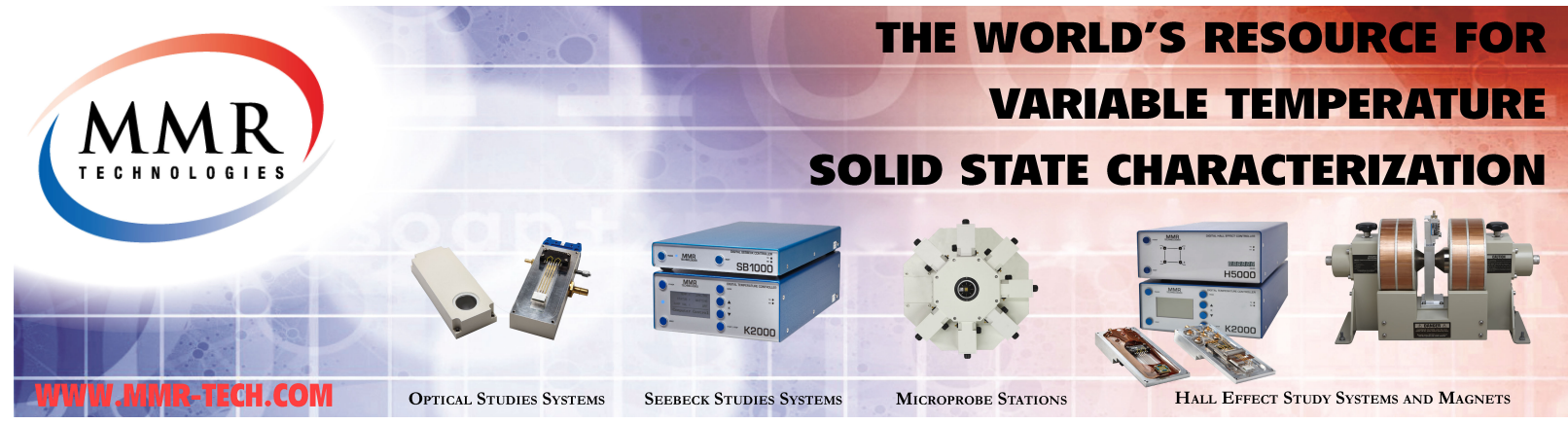




\title{
Crossover in thermal transport mechanism in nanocrystalline silicon
}

\author{
Arun Bodapati ${ }^{\mathrm{a})}$ and Pawel Keblinski ${ }^{\mathrm{b})}$ \\ Department of Materials Science and Engineering, Rensselaer Polytechnic Institute, Troy, \\ New York 12180
}

\author{
Patrick K. Schelling \\ Advanced Materials Processing and Analysis Center (AMPAC), Department of Physics, \\ University of Central Florida, Orlando, Florida 32816 \\ Simon R. Phillpot \\ Department of Materials Science and Engineering, University of Florida, Gainesville, Florida 32611
}

(Received 9 November 2005; accepted 7 March 2006; published online 4 April 2006)

\begin{abstract}
Using vibrational mode analysis, we demonstrate that lattice vibrations in small grain $(\leqq 3 \mathrm{~nm})$ structurally inhomogeneous nanocrystalline silicon are almost identical to those in homogeneous amorphous structures with the majority of the vibrations delocalized and unpolarized. As a consequence the principal thermal conductivity mechanism in such a nanocrystalline material is the same as in the amorphous material. With increasing grain size the ability of vibrations to homogenize over the nanocrystalline structure is gradually lost and the phonon spectrum becomes progressively more like that of a crystalline material; this is reflected in a crossover in the mechanism of thermal transport. (C) 2006 American Institute of Physics. [DOI: 10.1063/1.2192145]
\end{abstract}

As the grain size of polycrystalline materials is reduced, a competition develops between the tendency to remain polycrystalline with a grain size in the nanometer range, on the one hand, and the tendency to become amorphous, on the other. ${ }^{1,2}$ In this letter, for the case of $\mathrm{Si}$ we elucidate the nature of the accompanying transition in the vibrational behavior of polycrystalline materials from vibrations characteristic of a crystalline system to those characteristic of the amorphous material. Remarkably, this transition in vibrational signature is independent of the structural transition. In particular, our atomic-level simulations demonstrate that a nanocrystalline material that displays the structural signatures of a crystalline material can simultaneously display the vibrational and thermal transport signatures characteristic of an amorphous material.

As a model system that represents the key feature of structural inhomogeneity, we have chosen a one-dimensional periodic superlattice of silicon grain boundaries with atomic interactions described by the Stillinger-Weber potential. ${ }^{3}$ As a representative grain boundary (GB), we select a high-angle, high-energy twist boundary on the (001) plane (see Fig. 1). The simulation cells are periodic in all three directions and consist of two grains misoriented by a twist along the $z$ direction, separated by two GBs. GB superlattices have a cross-sectional area of $2.1 \times 2.1 \mathrm{~nm}^{2}$ and GB spacings $d_{z}$ (grain sizes) ranging from 1.6 to $5.4 \mathrm{~nm}$, with total number of atoms ranging from 696 to 2320 , respectively. The structures were first annealed by molecular dynamics (MD) simulations at $80 \%$ of the melting point of $\mathrm{Si}$, then quenched, and finally relaxed to zero temperature and zero pressure. ${ }^{4}$ The frequencies and relative displacements of each vibrational mode were calculated via diagonalization of the forceconstant matrix. ${ }^{5}$ The computational load of diagonalizing large matrices limited the largest grain size in our vibrational analyses to $5.4 \mathrm{~nm}$. For comparison, we also analyzed a su-

\footnotetext{
${ }^{\text {a) }}$ Electronic mail: bodapa@rpi.edu

b)Electronic mail: keblip@rpi.edu
}

perlattice of high-angle twist GB on the (111) plane, the so-called $\Sigma 31 \mathrm{~GB}$, which has GB energy about half that of the (001) GBs and the interface displays a much higher degree of structural order. All of the model GB structures are dominated by crystalline grain interiors, sandwiched by very narrow planar GB regions, as depicted in Fig. 1. The choice of these simplified nanocrystalline structures enables analyses of a clarity that would not be possible in a fully threedimensional nanostructure. It has the additional merit of computational tractability, particularly for the vibrational analyses.

In our analysis we compare the vibrational spectra of nanocrystalline superlattices with those characterizing amorphous and perfect crystal silicon. Phonons, i.e., vibrational waves in crystalline materials, are delocalized and characterized by a well-defined wave vector and polarization. Most vibrational modes in amorphous silicon are also delocalized; however, they do not have a well-defined wave vector or polarization and are better classified as diffusive modes, or "diffusons."6

Our model GB structures are structurally dominated by crystalline grain interiors and exhibit local vibrational den-

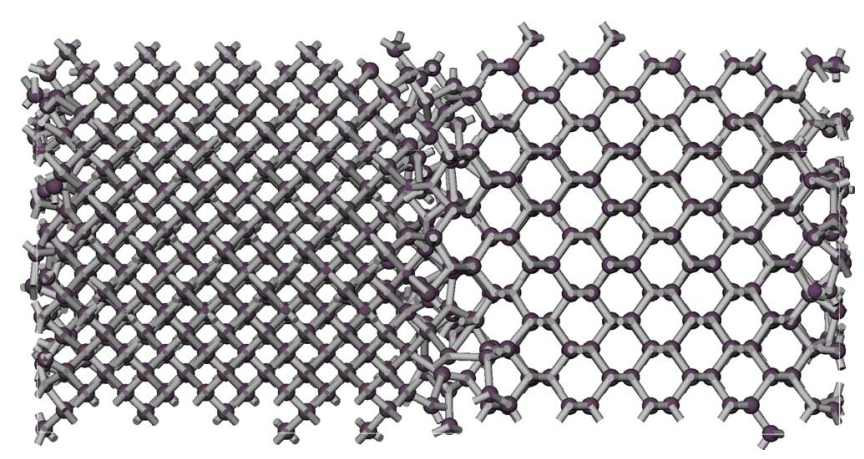

FIG. 1. (Color online) Atomic positions and bonding in a high-angle (001) $\Sigma 29$ twist grain boundary in silicon with GB separation $d_{z}=2.7 \mathrm{~nm}$. Two grains are separated by two GBs, one at the center and one at the edges of the simulation box. Periodic boundary conditions are used in all directions. 


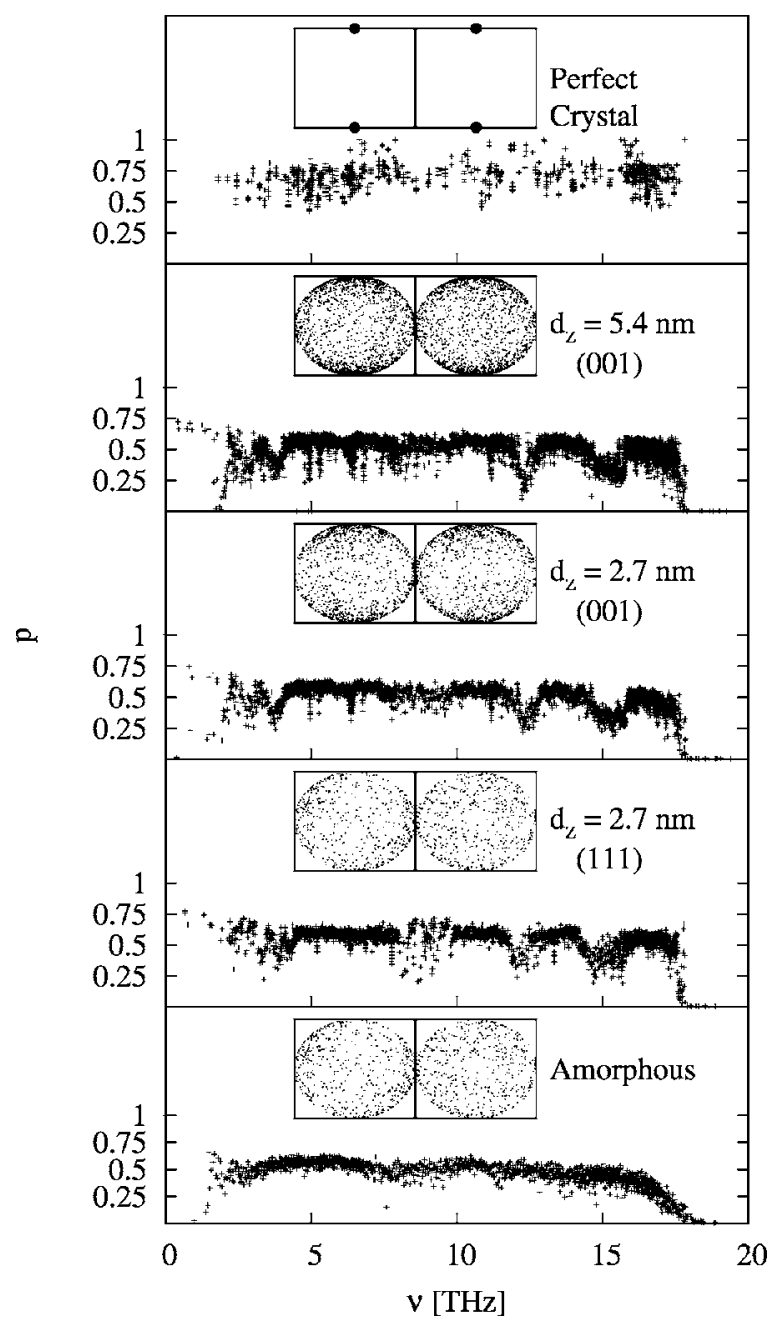

FIG. 2. The participation ratio vs frequency for amorphous silicon, $d_{z}=2.7 \mathrm{~nm}$ (111) GB and (001) GB, $d_{z}=5.4 \mathrm{~nm}$ (001) GB, and perfect crystal structures. Insets show projections of Cartesian components $z$ vs $x$ (left) and $z$ vs $y$ (right) of unit normalized polarization vectors for a typical mode around $3 \mathrm{THz}$ frequency.

sity of states (VDOS) (not shown) akin to that of bulk silicon, while the local VDOS at GB regions are akin to that of amorphous silicon. Similar behavior was observed in recent studies of amorphous/crystalline silicon sandwich structures. ${ }^{7}$

The degree of spatial localization of a vibrational mode can be quantified via the participation ratio, which measures the fraction of atoms participating in a given mode. ${ }^{5}$ According to Fig. 2, the amorphous, the two $d_{z}=2.7 \mathrm{~nm}$, and the $d_{z}=5.4 \mathrm{~nm}$ structures have similar localization characteristics, with most of the modes delocalized with participation ratios $\sim 0.5-0.6$. Furthermore, GB structures also have a number of high-frequency modes localized within clusters of only a few atoms, analogous to localized high-frequency modes in amorphous $\mathrm{Si}$. Since these frequencies are higher than any in the perfect crystal, they can be identified with localized vibrations at the grain boundaries. Interestingly, in the GB structures there are some low participation ratio modes at lower frequencies. These modes are mostly localized at GBs with a few populated in grain interiors; they will be described in detail elsewhere. Participation ratio for perfect crystal modes (see Fig. 2) is generally higher than for amorphous and nanocrystalline materials and all the modes are delocalized.
While the participation ratios for amorphous and nanocrystalline structures are qualitatively similar, the polarization characteristics are dramatically different. The insets in Fig. 2 show the projection of normalized unit polarization vectors onto circles for a typical mode at about $3 \mathrm{THz}$ frequency. Each point in the circle represents a Cartesian component of a unit polarization vector of an atom, for the given particular mode. For the amorphous and $d_{z}=2.7 \mathrm{~nm} \mathrm{~GB}$ (111) structures, the atomic displacements are uncorrelated and fill the projected polarization sphere more or less uniformly. However, it can be seen that for the $d_{z}=2.7 \mathrm{~nm}(001)$ GB structure, the mode has a small degree of preferential direction which becomes more pronounced for $d_{z}=5.4 \mathrm{~nm}$ (001) GB structure, exhibiting the clustering of unit vectors in certain regions of the unit sphere. With increasing grain separation, the modes begin to acquire longer spatial polarization memory, characteristic of phonons in a crystalline structure which have a well-defined polarization (top panel in Fig. 2).

Having demonstrated the close similarity in the nature of atomic vibrations between small grain size nanocrystalline materials and the corresponding amorphous materials we now explore consequences of these similarities on thermal transport. To expose the thermal transport mechanism we evaluate the thermal conductivity via two independent methods. The first method is based on a harmonic theory of disordered solids by Allen and Feldman ${ }^{8}$ (AF) and is applicable to systems with thermal transport dominated by delocalized and unpolarized vibrations, such as amorphous structures. Within this method the thermal conductivity $\kappa_{\mathrm{AF}}$ is given by

$$
\kappa_{\mathrm{AF}}=\frac{1}{V} \sum_{i} c_{i}(T) D_{i}
$$

where $V$ is volume, $c_{i}$ and $D_{i}$ are the mode heat capacity and diffusivity, respectively, and the sum is over all vibrational modes. The $D_{i}$ are calculated from elements of the heat current operator within the harmonic approximation. ${ }^{8}$ The AF theory is inapplicable if the heat is carried by polarized phonons, for which anharmonicity plays an important role, or if the structure is inhomogeneous. For our GB structures we focus our attention on mode diffusivities and the corresponding thermal conductivity along the direction normal to the GB plane. The second method to evaluate thermal conductivity is via direct MD simulations at room temperature, where a temperature gradient induced by a heat source and a heat sink, and the thermal conductivity are extracted from the slope of the linear temperature profile via Fourier's law. ${ }^{9}$ In these MD simulations to reduce any finite size effects ${ }^{10}$ we used GB superlattices consisting of 12 grains separated by 12 crystallographically identical GBs. The simulation cells were periodic in all three directions with a cross section of $2.1 \times 2.1 \mathrm{~nm}^{2}$ and the grain boundary spacing $d_{z}$ ranging from 1.6 to $10.8 \mathrm{~nm}$, with the corresponding total number of atoms ranging from 4176 to 27840 . For small grain structures temperature profiles were linear, while for larger grain structures we observed incipience of temperature discontinuities at GBs. In all cases we report overall thermal conductivity obtained from the average temperature gradients.

Grain boundaries in our nanocrystalline structures limit the thermal transport. Therefore in Fig. 3, rather than the thermal conductivity itself, we plot the ratio of thermal conductivity to the separation between grains in (001) GB struc- 


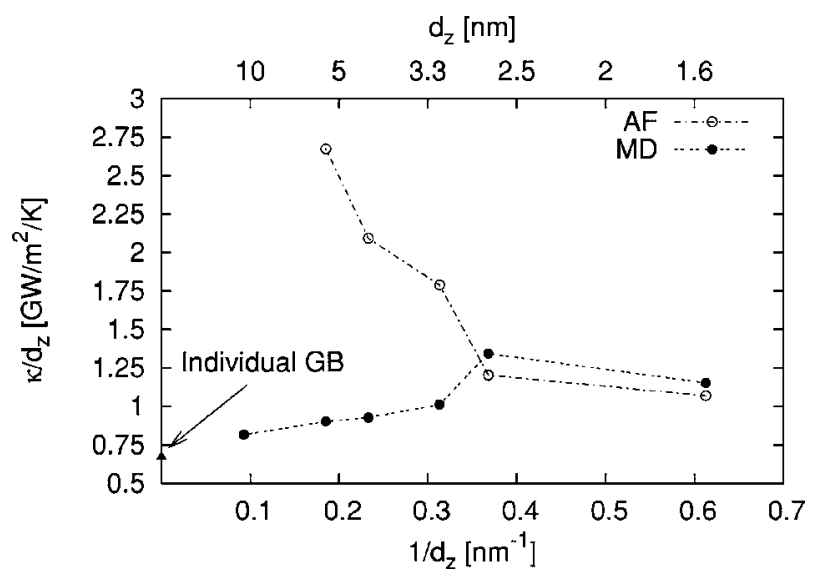

FIG. 3. Thermal conductivity in the direction normal to the GB plane divided by the GB separation, which is the nominal GB conductance, as a function of $1 / d_{z}$. Two data sets are for thermal conductivity obtained in MD simulations and from the Allen-Feldman theory of disordered solids. Individual GB conductance of GB (001) is shown at $1 / d_{z}=0$.

tures, $\kappa / d_{z}$, which gives the nominal value of the GB interfacial conductance $G_{\mathrm{GB}}{ }^{11}$ Also, for more compact data presentation we plot $\kappa / d_{z}$ as a function of $1 / d_{z}$ rather than $d_{z}$ itself. Since the MD simulations are classical, we compare MD with AF calculated with the classical heat capacity. As shown in Fig. 3, for $d_{z} \leqslant 3 \mathrm{~nm}, \kappa_{\mathrm{AF}} \sim \kappa_{\mathrm{MD}}$, which demonstrates that the harmonic theory of disordered, homogenous solids describes well thermal transport properties of small grain size nanocrystalline material. This finding is consistent with the previously described vibrational analysis. For reference we note that for our amorphous structure $\kappa_{\mathrm{AF}} \approx \kappa_{\mathrm{MD}}$ $\approx 0.85 \mathrm{Wm}^{-1} \mathrm{~K}^{-1}$.

Increasing the grain size above $\sim 3 \mathrm{~nm}$ leads to a decrease of the nominal grain boundary conductance $\kappa / d_{z}=G_{\mathrm{GB}}$, obtained from MD simulations, while $G_{\mathrm{GB}} \mathrm{ob}-$ tained from the theory of disordered solids increases with increasing grain size. This demonstrates that, as expected, the picture of thermal energy as being carried by unpolarized vibrations is not applicable for larger grain nanocrystals. We also used MD simulations to evaluate the GB conductance of individual GBs (i.e., widely separated GBs; see Ref. 10 for more details). This GB conductance was $G_{(001)}=0.67 \mathrm{GW} \mathrm{m}^{-2} \mathrm{~K}^{-1}$ (see Fig. 3). Figure 3 demonstrates that with increasing GB separation, the nominal gain boundary conductance tends towards the value characterizing individual GB conductance. This and the polarization analysis (see insets in Fig. 2) suggest that large grain size nanocrystalline materials exhibit a thermal transport mechanism akin to that present in large grain size polycrystalline material, namely, the heat is carried by polarized and propagating phonons that are scattered by GBs. Furthermore, from Fig. 3 it is apparent that there is a continuous but distinct crossover in the thermal transport mechanism with increasing grain size from diffuse transport in nanocrystalline structures to a more conventional phonon transport in larger polycrystalline structures.

Finally, we comment on the expected temperature dependence of the thermal conductivity of nanocrystalline silicon. For small grain sizes, we demonstrated that the thermal transport is akin to that present in amorphous silicon; therefore, we expect that thermal conductivity will increase with temperature, as is the case for amorphous silicon. ${ }^{12,13}$ By contrast, crystalline silicon, other than at very low temperature, shows a decrease of thermal conductivity with increasing temperature due to anharmonicity related phononphonon thermal scattering. ${ }^{14}$ In the case of our studies even the largest grain sizes of $\sim 10 \mathrm{~nm}$ are significantly smaller than the perfect crystal phonon mean free path. ${ }^{9}$ Consequently thermal conductivity and its temperature dependence in nanocrystalline silicon, even with relatively large grain sizes, will be controlled by the GB conductance. The silicon GB conductance was demonstrated to increase with increasing temperature $;^{10}$ therefore, we expect that nanocrystalline $\mathrm{Si}$ will exhibit increase in thermal conductivity with increasing temperature. In fact such behavior was observed in experimental studies of nanocrystalline silicon with the average grain size of $\sim 200 \mathrm{~nm}^{15}$

In summary we have demonstrated that small grain nanocrystalline structures, despite being structurally inhomogeneous, are seen by vibrations as homogeneous structures. Increasing the grain size leads to gradual recognition by the lattice vibrations that the structure is indeed inhomogeneous. This transition in the nature of the vibrations has a corresponding transition in the mechanism of the heat transport from one akin to that exhibited by amorphous material to that characterizing polycrystalline material.

${ }^{1}$ S. Veprek, Z. Iqbal, and F. A. Sarott, Philos. Mag. B 45, 137 (1982).

${ }^{2}$ D. Wolf, J. Wang, S. R. Phillpot, and H. Gleiter, Phys. Lett. A 205, 274 (1995).

${ }^{3}$ F. H. Stillinger and T. A. Weber, Phys. Rev. B 31, 5262 (1985).

${ }^{4}$ P. Keblinski, S. R. Phillpot, D. Wolf, and H. Gleiter, J. Am. Ceram. Soc. 80, 717 (1997).

${ }^{5}$ P. K. Schelling and S. R. Phillpot, J. Am. Ceram. Soc. 84, 2997 (2001).

${ }^{6}$ P. B. Allen, J. L. Feldman, J. Fabian, and F. Wooten, Philos. Mag. B 79, 1715 (1999).

${ }^{7}$ J. L. Feldman and N. Bernstein, Phys. Rev. B 70, 235214 (2004).

${ }^{8}$ P. B. Allen and J. L. Feldman, Phys. Rev. B 48, 12581 (1993).

${ }^{9}$ P. K. Schelling, S. R. Phillpot, and P. Keblinski, Phys. Rev. B 65, 144306 (2002).

${ }^{10}$ P. K. Schelling, S. R. Phillpot, and P. Keblinski, J. Appl. Phys. 95, 6082 (2004)

${ }^{11}$ E. T. Swartz and R. O. Pohl, Rev. Mod. Phys. 61, 605 (1989).

${ }^{12}$ J. L. Feldman, M. D. Kluge, P. B. Allen, and F. Wooten, Phys. Rev. B 48, 12589 (1993).

${ }^{13}$ D. G. Cahill and R. O. Pohl, Phys. Rev. B 35, 4067 (1987).

${ }^{14}$ C. J. Glassbrenner and G. A. Slack, Phys. Rev. 134, A1058 (1964).

${ }^{15}$ S. Uma, A. D. McConnell, M. Ashegi, K. Kurbayashi, and K. E. Goodson, Int. J. Thermophys. 22, 605 (2001). 